\title{
XXIV. Facts and observations relating to the science of phonetics
}

\section{R.G. Latham}

To cite this article: R.G. Latham (1841) XXIV. Facts and observations relating to the science of phonetics, Philosophical Magazine Series 3, 18:115, 124-130, DOI: 10.1080/14786444108650259

To link to this article: http://dx.doi.org/10.1080/14786444108650259

曲 Published online: 01 Jun 2009.

Submit your article to this journal

Џ Article views: 2

Q View related articles $₫$ 
and burns with a strong yellow flame, emitting much smoke. It boils at $168^{\circ} \mathrm{C}$. Its specific gravity is 1.1006 at $27^{\circ} \mathrm{C}$. It is pretty soluble in water, and much more so in alcohol and æether. Potassium effervesces strongly when placed in it, but neither the alcoholic nor aqueous solution of potash has any effect upon it. Solid potash, with the assistance of heat, converts it into a brown resin. It dissolves iodine very easily, hut without any violent action even when heated. In the cold muriatic acid gives it a fine red colour, and with the assistance of heat turns it into a dark brown resin. Nitric acid first reddens, then chars it. Sulphuric acid has a similar effect.

The results of its analyses are as follows:

I. 0.330 substance gave 0.74 .7 carbonic acid, and 0.130 water.

11. 0.324 gave 0.725 carbonic acid, and 0.1275 water.

III. 0.3185 gave 0.7205 carbonic acid, and 0.128 water.

Found.

$$
\text { I. }
$$



Carbon ... 62.59

Hydrogen 4.37

Oxygen... 33.04

This gives the formula:

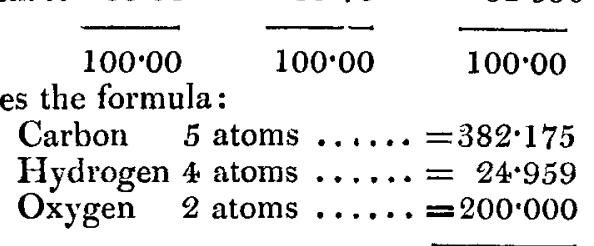

$$
\text { Atomic weight } 607 \cdot 134
$$

Calculated.

$62 \cdot 94$

$4 \cdot 11$

$32 \cdot 94$

$99 \cdot 99$

It is evident that this oil has a very different composition from oils in general, as it contains oxygen and hydrogen only in the proportions to form water, while all other known oils contain a great excess of hydrogen.

XXIV. Facts and Observations relating to the Science of Phonetics. By R. G. Latham, Fellow of King's College, Cambridge.*

$$
\begin{gathered}
\text { PanT I.-Upon the Aspirates (real and accredited) of } \mathrm{K} \\
\text { and } \mathrm{G} \text {. }
\end{gathered}
$$

THE object of the present paper is to show that the real aspirates of $K$ and $G$ are not the sounds that are generally considered as such; in other words, that they are

* Communicated by the Author. 
not the powers of the German $c h$ and Scotch gh, but that they are sounds perfectly distinct from either the one or the other of those articulations.

The question in point, besides its value in etymology, has a value in acoustics; at least in that particular part of them, that deals with the affinities and analogies of articulate sounds.

This part of acoustics may conveniently be called the province of phonetics.

How far the articulate sounds are systematically related to each other; how far and in what cases they run into each other; how far the chain of relationship is lineal, and how far it is circular; how far vowels and consonants, mutes and liquids differ in kind from each other; these questions, and questions similar to these, constitute the province of phonetics.

These investigations must be distinguished, on the one hand, from those of physiology, and on the other hand, from those of etymology.

The science of phonetics determines how two given articulations are related; the science of physiology inquires how they are produced.

Phonetics tell us that between two articulations an immutable and essential relation exists; whilst etymology observes that under certain circumstances one articulation is changed into another. Very often etymology does more; it assumes the alliance from the change.

Now it does not follow, in etymology, that because in a given language one sound changes to another, those two sounds are, therefore, naturally allied to each other; although such is often (far, however, from always) the case.

Nor yet has it been proved in physiology that the sounds which to the ear sound alike, are produced by a like disposition of the parts of the mouth or larynx; although that such a correspondence exists is highly probable.

The truth is, that the special study of phonetics, instead of being promoted by the grammarian and anatomist, has been retarded by them. Etymological and physiological tests, etymological and physiological classifications, have been applied where acoustic principles alone ought to have been recognized. A necessary correspondence, moreover, between the three kinds of sciences, which in grammar may be proved nonexistent, and in physiology has not been proved at all, has for the most part been gratuitously assumed; and more than this, in those cases where the three tests have not coincided, the disposition has been to sacrifice the phonetic test to the other two. 
The Phonetic test stands thus-Two sounds are allied, because, to the ear, they sound alike.

The physiological test thus--Two sounds are allied, because, in the mouth and larynx, they are formed alike.

The etymological test thus-Two sounds are allied, because, under certain circumstances, one is changed to the other.

Expressed in Latin axioms, the etymological test is, Propter fortunas articulatio est id quod est; the physiological one, Propter formationem articulatio est id quod est; and, finally, the phonetic one is, Propter sonum articulatio est id quod est. The coincidence of the three is (in the present state of our knowledge) to be considered as an accident.

Phonetic science considers sounds irrespective of the signs by which they are expressed, and irrespective of the names by which they are called. The sound of the $b$ (in $b a t$ ) is the same, whether it be expressed by the sign $b$ or $\beta$; it is also the same whether it be called bee or beta. A sound is not double because it is spelt with two letters, nor yet single because it is expressed by one. The th in thin is a simple sound, irregularly expressed: the $x$ in box is a double one compendiously spelt. In questions of the kind in point, the eye has so often misled the ear that the above given truisms are scarcely to be considered superfluous. In points of acoustics the blindest guides are the best.

To guard against the influence of names and signs it is convenient to express the relations of certain sounds, arithmetically; and besides this, in the case of such simple single sounds as have no simple single sign (or letter) to denote them, to coin or borrow appropriate signs as the occasion requires.

The subjoined scales of relationship being those that are generally recognized, it is unnecessary to go beyond the mere exhibition of them.

The sound of $b$ (in $b i n$ ) is in a certain relation to the sound of $p$ (in $p i n)$. Now,

$$
\begin{aligned}
& \mathrm{v}: f:: \mathrm{b}: \mathrm{p} \text {. and } \\
& \mathrm{d}: \mathrm{t}:: \mathrm{v}: \mathrm{f} \text {. and } \\
& \gamma^{1}: \mathrm{p}^{2}:: \mathrm{d}: \mathrm{t} \text {. and } \\
& \mathrm{g}: \mathrm{k}:: \delta: \mathrm{g} \text {. and } \\
& z: \mathrm{s}:: \mathrm{g}: \mathrm{k} \text {. and } \\
& \zeta^{3}: \sigma^{4}:: \mathrm{z}: \mathrm{s} .
\end{aligned}
$$

${ }^{2}$ The th in thine. ${ }^{2}$ The $t h$ in thin. ${ }^{3}$ The $z$ in azure. $\quad 4 s h$ in shin. 
Again, the sound of $f($ as in $f a)$ is in a certain relation to the sound of $p$ (as in $p a$ ); or, expressed more loosely, $f$ is in a certain relation to $p$. Now

$$
\begin{aligned}
& \mathrm{v}: \mathrm{b}:: \mathrm{f}: \mathrm{p} \text {. and } \\
& \mathrm{p}: \mathrm{t}:: \mathrm{v}: \mathrm{b} \text {. and } \\
& \gamma: \mathrm{d}:: \mathrm{p}: \mathrm{t} \text {. and } \\
& \sigma: \mathrm{s}:: \mathrm{\gamma}: \mathrm{d} \text {. and } \\
& \xi: \mathrm{z}:: \sigma: \mathrm{s} \text {. }
\end{aligned}
$$

To these two classes of relation the names Hard and Soft, Lene and Aspirate may (for want of better) be allowed to stand; so that the double relationship is expressed in the following table:-

Lene.

Hard. Soft.

$p \cdot b$

$t \cdot d$

l. g

$s \cdot z$

or

Hard

Lene. Aspirate.

$\begin{array}{ll}p \cdot f & f \\ t & \mathrm{p} \\ k & ? \\ s & =\end{array}$
Aspirate.

Hard. Soft.

$$
\begin{aligned}
& f \cdot v \\
& \dot{p} \cdot v \\
& ? \cdot \frac{\gamma}{\sigma} \cdot \zeta
\end{aligned}
$$

$$
\text { Soft. }
$$

Lene. Aspirate.

$$
\begin{array}{lll}
b & \cdot & v \\
d & \cdot & \delta . \\
g & \cdot & ? \\
z & \cdot & \zeta
\end{array}
$$

Here we have (so to say) four parallel lines of double relationship. The sequence, however, although perfect as far as it goes, is incomplete. We miss the sounds that are to $k$ and $g$, as $f$ is to $b$ and $v$ to $b, \& c$. \&c. Were it not for this deficiency we should have a mute-system of sixteen allied articulations, square and symmetrical, each articulation being to the other doubly related, i.e. hard or soft, lene or aspirate. As it has been said before, it is the object of the present paper to fill up the deficiency in question, and, subordinately to the main end, to show that the sounds that are considered to be the aspirates of $k$ and $g$ are not so in reality. The sounds that in all the works (philological or physiological) with which I am acquainted, have been supposed to hold the relationship in point, are the German ch (as in auch) and the Gaelic gh (as in lough) respectively. Let these two sounds be expressed by $k h$ and $g h$ respectively, and let the undetermined aspirates of $k$ and $g$ be expressed by $\mathbf{K}_{\mathrm{x}}$ and $\mathbf{G}_{\mathrm{x}}$ respectively.

The affinities which invalidate the current opinion, are, first, the affinity which exists, and which is recognized, between the mute sounds of $b$, and $v$ and the semivowel sound of $w$; and secondly, the affinity (existing and recognized) be- 
tween the semivowel sounds of $y$ (as in $y a$ ) and the mute sound of $g$ (as in $g a$ ).

The sound of $w$ (as in $w a$ ) is to the sound of $v$ (as in $v a$ ) in a certain degree of relation; and, through this, it is allied to $b$ (as in $b a$ ), in a manner determinable by the relation of $v$ and $b$ : and, again, $y$ (as in $y a$ ) is to $g$ (as in $g a$ ) as $w$ is to $v$. Hence it arises that the sound which shall be to $g$ as $v$ is to $b$, shall be to $g$ and $y$ as $v$ is to $b$ and $w$. Expressed formally, the fact stands thus :

$$
\mathrm{G}_{\mathrm{x}}: g \text { and } y:: v: b \text { and } w \text {. }
$$

In a short tract, published in 1835 , I stated that the sound in point must be on the " $y$-side of $g$," and that, although a Iudicrous mode of speaking, expressed my meaning fully.

The conditions then of $\mathrm{G}_{\mathrm{x}}$ are, that it must be in a certain relation to $g$ and $y$; in which relation $I$ an satisfied that $g h$ is not.

It may not be thought superfluous to remark, that the two last assertions, that is, the assumptions of the question in hand, are made irrespective of any previous reasoning. I consider that they rest upon the evidence of our sense of hearing. Expressed formally, the facts and inference are as follows:

$$
\mathbf{G}_{\mathbf{x}}: g \text { and } y:: v: b \text { and } w \text {. which } g / h \text { is not. }
$$$$
\text { * * * * * * }
$$

What $g h$ is, is another question. Unless the number of mutes is to be reduced to four, sounds like $b, p, f, v, \& c$. \&c. must be considered not as varieties of a given typical sound, but as sounds specifically distinct: and, again, unless the number of mutes is to be indefinitely increased, sounds like the $t$ and $d$ cerebral of the Hindoos (I speak in the way of illustration) must be considered, not as sounds specifically distinct, but as varieties of a given typical sound.

To come, then, under the necessary conditions, the sound of $\mathrm{G}_{\mathrm{x}}$ must be specifically different from that of $G$. Now $G h$ (as may be shown at another time) is no specific sound, but only a variety.

The force of the worls specific and variational depends upon their definitions. To say that $b$ is a variety of $t$ is true or false, according to the definition of the term variety; and the assertion is objectionable only on the score of inconvenience. To say, however, that $b$ is a variety of $s$ (as is often said by those who are strangers to the sound) involves something more than an inconvenient definition. It is an error in respect to the fact.

Having (to my own satisfaction at least) inferred that $G / h$ 
was not $G_{x}$, I was left to consider what really was $G_{x}$, and whether such a sound either actually existed or was capable of existing.

I drew the distinction between the actual and the possible existence of a sound for the following reason. It is very evident that although an articulation may be capable of being both formed by the larynx and distinguished by the ear, it may not exist in any spoken language; in other words, it does not follow that because a sound exists in posse it exists in esse also. If the French were the universal language, the sound of the English $t h$ would exist only in the capabilities of the ear and larynx; it would be a sound in posse, or a latent sound.

Now against $G_{x}$ being a possible sound, I knew of no reason except the accident of its not being found in any of the well-known languages; whilst in favour of its being one were the analogies of the other mutes. This latter fact having convinced me that the sound in point was within the compass of the vocal organs, I conceived it possible that (two facts being ascertained) it might be formed $a^{2}$ prior $i$, that is, independent of imitation. These two facts were, first, the determination of the difference between an aspirate and its lene in respect to their sounds; secondly, the determination of the same in respect to the disposition of the parts of the mouth and larynx. In the midst of my investigations an accident intervened, that solved all the difficulties. I found, in Rask's Laplandic Grammar, the description of a rare and peculiar sound. It was not $G h$; nor yet was it either $G$ or $Y$. It was something between the two. This threw a light upon the question; so that all that now remained for me to do, was to hear the sound. In the town where I was residing there was a Laplander from Norwegian Finmark, whom the Norse government were instructing in reading and writing. Having sent for this person, and having set before him the words that Rask had given as examples, I found that his pronunciation of the sound in point coincided with the description of Rask, with my own previous notions as to its nature, and, consequently, with the conditions of $G_{x}$; in other words, the Lappish $z$ (so expressed in Rask's Grammar) was $\mathbf{G}_{\mathbf{x}}$.

Now, in the foregoing notices, nothing has been said concerning $K$ and $K_{\mathrm{x}}$. Nothing has been said concerning them, because it is so evident that they follow the analogies of $G$ and $G_{x}$, that any mention of them would have been superfluous. 'The existence in posse of $\mathrm{K}_{\mathrm{x}} \mathrm{I}$ consider to be all but demonstrated; of its existence in esse $I$ am less certain. It is probable that in some of the Greek dialects $k$ is pronounced as $\mathrm{K}_{\mathrm{x}}$.

Phil. Mag. S. 3. Vol. 18. No. 115. Feb. 1841, K 
In certain dialects of the same language $I$ imagine that $\gamma=G_{x}$. The letter $g$ in the mouth of a native of Berlin, is pronounced (as I am told) intermediately to $G$ and $Y$.* If this be true, it is in all probability $\mathbf{G}_{\mathbf{x}}$. It is probable that in certain dialects, or in certain stages of the Anglo-Saxon, $\zeta$ has been sounded as $\mathbf{G}_{\mathbf{x}}$.

$K_{x}$ then being represented by $x$, and $G_{x}$ by $\gamma$, we have, as an exhibition of the system formed by the sixteen mutes, the following table of double relations.

\begin{tabular}{|c|c|c|c|}
\hline \multicolumn{2}{|c|}{ Lene. } & \multicolumn{2}{|c|}{ Aspirate. } \\
\hline Hard & Soft. & Hard. & Soft. \\
\hline$\underset{t}{p}$ & $\cdot \begin{array}{ll}b & b \\
\cdot & d\end{array}$. & $f$ & $\begin{array}{l}v . \\
\delta .\end{array}$ \\
\hline$k$ & - $g$. & $x$ & \\
\hline$s$ & $z$ & $\sigma$ & \\
\hline
\end{tabular}

I consider that these relations are immutable and essential, independent of the physiological arrangement, in their formation, of the parts of the mouth and larynx, and independent of their interchanges in the grammars of particular languages. I consider, moreover, that the number of specifically distinct mutes, independent of the fact of their being found in languages, is neither more nor less than sixteen, and that, in the present state of our knowledge, there is no more convenient mode of distinguishing mutes from other articulations, than by saying that every mute is one of four sounds, each of which forms a part of a system of four, and each of which is to some other hard or soft, and lene or aspirate.

XXV. Reply to Mr. Airy's Remarks on Professor Challis's Investigation of the Motion of a small Sphere vibrating in a resisting Medium. By the Rev. J. Challis, Plumian Professor of Astronomy in the University of Cambridge.

To the Editors of the Philosophical Magazine and Journal. Gentlemen,

A LLOW me, through the medium of your Journal, to express my thanks to Mr. Airy for calling my attention, by his letter in your Supplementary Number, to a step in my solution of the problem of the resistance to a sphere vibrating in an elastic medium, which I had left unexplained. I have certainly considered it possible that the velocity of the fluid at a given distance from the centre to or from which it is directed may, at a given instant, be different in different directions from the centre, provided there be no abrupt variation.

[* The letter $g$, at least when final, is precisely so pronounced by the Swedes.-EDrt.] 